\title{
Choosing Our Words: Retrieval and Selection Processes Recruit Shared Neural Substrates in Left Ventrolateral Prefrontal Cortex
}

\author{
Hannah R. Snyder, Marie T. Banich, and Yuko Munakata
}

\begin{abstract}
When we speak, we constantly retrieve and select words for production in the face of multiple possible alternatives. Our ability to respond in such underdetermined situations is supported by left ventrolateral prefrontal cortical (VLPFC) regions, but there is active debate about whether these regions support (1) selection between competing alternatives, (2) controlled retrieval from semantic memory, or (3) selection and controlled retrieval in distinct subregions of VLPFC (selection in mid-VLPFC and controlled retrieval in anterior VLPFC). Each of these theories has been supported by some prior evidence but challenged by other findings, leaving the debate unresolved. We propose that these discrepancies in the previous literature reflect prob-
\end{abstract}

\section{INTRODUCTION}

One of the defining characteristics of human intelligence is the ability to respond flexibly to the environment. Rather than being tied to habitual responses, we are able to respond to a given environmental context in a wide variety of ways, informed by past experience, current context, and long-term goals. This ability is arguably what allows us to engage in an almost infinite repertoire of behaviors, including the most human behavior of all, language. During ordinary language production, words must constantly be retrieved and selected for production in the face of multiple possible alternatives. For example, when constructing a sentence, a speaker must not only choose the intended message but must also retrieve and select among multiple syntactic structures and words which are all compatible with the intended message. There is broad consensus that our ability to respond in such underdetermined situations depends on cognitive control processes subserved by left ventrolateral prefrontal cortical (VLPFC) regions. However, exactly what this neural region does to support these fundamental cognitive processes is strongly debated.

Three competing theories have been proposed, positing that left VLPFC subserves (1) selection among competing alternatives (e.g., Botvinick, Braver, Barch, Carter, \& Cohen, 2001; Thompson-Schill, D’Esposito, Aguirre, \&

University of Colorado at Boulder lems in the way that selection and controlled retrieval processes have been operationalized and measured. Using improved measures, we find that shared neural substrates in left VLPFC support both selection and controlled retrieval, with no dissociation between mid and anterior regions. Moreover, selection and retrieval demands interact in left VLPFC, such that selection effects are greatest when retrieval demands are low, consistent with prior behavioral findings. These findings enable a synthesis and reinterpretation of prior evidence and suggest that the ability to respond in underdetermined situations is affected by both selection and retrieval mechanisms for verbal material subserved by left VLPFC, and these processes interact in meaningful ways.

Farah, 1997), (2) controlled retrieval from semantic memory (e.g., Martin \& Cheng, 2006; Wagner, Paré-Blagoev, Clark, \& Poldrack, 2001), or (3) selection and controlled retrieval in different regions of the VLPFC (selection in mid-VLPFC and controlled retrieval in anterior VLPFC; e.g., Badre \& Wagner, 2007; Badre, Poldrack, Paré-Blagoev, Insler, \& Wagner, 2005). Although each of these theories has been supported by some prior evidence, each has also been challenged by other findings. Resolving this debate will advance our understanding of language production, in addition to speaking to broader issues about the nature of the functional organization of PFC, and the neural bases for specializations of distinct subregions (e.g., Petrides, 2005; Duncan \& Owen, 2000; Miller, 2000).

According to the selection hypothesis, left VLPFC resolves competition between multiple automatically activated representations to select a single response for output (e.g., Botvinick et al., 2001; Thompson-Schill et al., 1997). The less differentiated the activation pattern across all possible responses, the more difficult it is to resolve the competition and the greater is the activation of left VLPFC (e.g., Thompson-Schill \& Botvinick, 2006). This hypothesis is supported by evidence that left VLPFC is recruited in situations requiring selection between multiple competing representations. For example, in the frequently used verb generation task, participants are instructed to say the first verb that comes to mind associated with a presented noun. Left VLPFC is more active when participants generate verbs 
for nouns with multiple verb associates (e.g., ball, associated with kick, hit, throw, etc.) versus one associate (e.g., scissors, associated with cut; Nelson, Reuter-Lorenz, Persson, Sylvester, \& Jonides, 2009; Nagel, Schumacher, Goebel, \& D’Esposito, 2008; Persson et al., 2004; Barch, Braver, Sabb, \& Noll, 2000; Thompson-Schill et al., 1997). In addition, left VLPFC is more active, and responses are slowed when people name pictures with low versus high name agreement (Kan, Kable, Van Scoyoc, Chatterjee, \& ThompsonSchill, 2006; Kan \& Thompson-Schill, 2004), generate items from larger categories (e.g., flower) than from smaller categories (e.g., red flower; Tremblay \& Gracco, 2006), and select a specific attribute to match words (e.g., color) versus matching on overall similarity (Snyder, Feigenson, \& Thompson-Schill, 2007).

Complimenting these findings, patients with left PFC damage are more impaired on generating verbs for nouns with many associates and then with a single associate (Tippett, Gendall, Farah, \& Thompson-Schill, 2004; Thompson-Schill et al., 1998). In addition, patients with left PFC damage have severely impaired spontaneous speech and verbal fluency but preserved naming (Robinson, Shallice, \& Cipolotti, 2006; Robinson \& Cipolotti, 2004; Randolph, Bruan, Goldberg, \& Chase, 1993). These patterns suggest that these patients are able to perform well when the response is well constrained by the stimulus but experience difficulty when selection demands are high.

In contrast, according to the controlled retrieval hypothesis, left VLPFC retrieves responses from semantic memory when such responses are effortful and require cognitive control (e.g., Martin \& Cheng, 2006; Wagner et al., 2001). Thus, the weaker the connection between the stimulus and the most accessible response (association strength), the more difficult it is to retrieve a response and the greater is the activation of the left VLPFC (e.g., Martin \& Cheng, 2006; Wagner et al., 2001). This hypothesis is supported by evidence that the left VLPFC is recruited when it is necessary to retrieve a weakly associated response. For example, the left VLPFC is more active and responses are slowed when participants generate verbs for nouns with weak versus strong verb associates (Crescentini, Shallice, \& Macaluso, 2010; Martin \& Cheng, 2006), make semantic relatedness judgments about weakly associated (compared with strongly associated) words (Chou, Chen, Wu, \& Booth, 2009; Chou, Booth, Bitan, et al., 2006; Chou, Booth, Burman, et al., 2006; Badre et al., 2005; Wagner et al., 2001), or retrieve information about briefly studied (vs. well-studied) items (Souza, Donohue, \& Bunge, 2009; Velanova et al., 2003). Consistent with this neuroimaging evidence, a patient with a left VLPFC damage has been reported to have impaired retrieval of weakly associated (but not strongly associated) verbs (Martin \& Cheng, 2006).

Recently, a synthesis of the controlled retrieval and selection hypotheses has been proposed (the two-process account; Badre \& Wagner, 2007; Badre et al., 2005; cf. Gold et al., 2006), positing that the left anterior VLPFC (BA 47) supports controlled retrieval of semantic knowledge from posterior conceptual stores, whereas the left mid-VLPFC (BA 45) supports postretrieval selection between active representations, irrespective of whether they were retrieved in an automatic or controlled manner. Suggestive evidence is provided by a review of the literature that reported peak coordinates in left anterior or mid-VLPFC in six studies identified as manipulating controlled retrieval and/or selection demands ${ }^{1}$ (Badre \& Wagner, 2007). This review found that putative selection manipulations tended to activate left mid-VLPFC whereas putative retrieval manipulations tended to activate left anterior VLPFC.

However, the purity of the manipulations of selection and retrieval demands in these studies are debatable. For example, one of the included studies found that mid-VLPFC was more active for task-switch than task-repeat trials (Badre \& Wagner, 2006), with the need to switch characterized as solely a manipulation of selection demands (because the old and new task rules compete; Badre \& Wagner, 2007). However, many theories of task-switching argue that switching also requires retrieving the new rule from memory (e.g., Altmann \& Gray, 2008; Miyake, Emerson, Padilla, $\&$ Ahn, 2004). Thus, although this evidence could be interpreted as consistent with the two-process account, it could be reinterpreted as consistent with other accounts as well.

Relatively few studies have directly tested this twoprocess account by manipulating selection and retrieval demands within the same experiment, and those studies have yielded inconsistent results. Only one study has found evidence suggesting differential responses to retrieval and selection demands in the left anterior and mid-VLPFC, respectively: In a lexical decision task, the left anterior VLPFC was more active for unprimed (thus harder to retrieve) than primed (easier to retrieve) words, whereas the mid-VLPFC was more active for words proceeded with an unrelated prime (presumably introducing competition) than unprimed words (Gold et al., 2006). However, Region $\times$ Condition interactions were not tested; thus, it is not clear if there is a full dissociation between these regions (as opposed to them supporting similar processes, with one contrast just failing to reach significance within each region, for example).

Three other experiments provide mixed results, finding selection or controlled retrieval in one or both regions of left VLPFC. In one experiment, participants decided which of two words was semantically related to a probe word: The left anterior VLPFC was specifically sensitive to the probe-target association strength (retrieval demand), whereas both the left anterior and mid-VLPFCs were more active when participants had to make a judgment based on a specific semantic feature (e.g., color, high selection demand) versus overall similarity (low selection demand; Badre et al., 2005). Likewise, in a task in which participants retrieved meanings of street signs, the left anterior VLPFC was more active for newly learned (high retrieval demand) than well-learned (low retrieval demand) meanings, whereas the left mid-VLPFC was sensitive to both retrieval demands and selection demands (one vs. two sign meanings; 
Souza et al., 2009). Finally, in the verb generation task and a noun generation variant (Crescentini et al., 2010), manipulations of controlled retrieval and selection were interpreted as yielding but did not clearly indicate a dissociation between mid-VLPFC and anterior VLPFC. Specifically, although left mid-VLPFC was sensitive to selection demands and left anterior VLPFC was sensitive to retrieval demands, each region also showed trends for sensitivity to the other demand, and Region $\times$ Condition interactions were either nonsignificant (verb generation task) or not tested (noun generation task). Moreover, the manipulation of retrieval demands seemed problematic, yielding effects in the noun generation task in the opposite direction from predictions (with greater activation in VLPFC when retrieval demands were low) and yielding no effects in the whole-brain analysis. Thus, the results are difficult to interpret and do not clearly indicate a dissociation between mid-VLPFC and anterior VLPFC.

Given this conflicting and inconclusive evidence, the debate about these fundamental cognitive processes and the neural substrates that subserve them is unresolved. In the current study, we use purer measures of selection and retrieval demand (Snyder \& Munakata, 2008), which allow us to examine these processes and their interaction in a way that was not previously possible. Specifically, prior studies of selection and retrieval during language production used measures of retrieval demand (association strength) and selection demand (competition) that were highly correlated, preventing clean separation of these factors. Association strength was calculated in terms of agreement, the proportion of a norming sample giving the most frequent response, and competition was calculated in terms of ratio, the proportion giving the most frequent response over the proportion giving the second most frequent response. Conditions differing on one measure also differed on the other, confounding retrieval and selection demands.

This problem can be addressed using new measures based on latent semantic analyses (LSA) that unconfound retrieval and selection demands and better capture the underlying theoretical constructs of association strength and competition (Snyder \& Munakata, 2008). LSA is a technique for extracting the similarity of words by analyzing large bodies of text, capturing contextual as well as cooccurrence information (Landauer, Foltz, \& Laham, 1998). LSA association values are absolute, that is, the association strength between any given stimulus and response is independent of alternative responses, consistent with characterizations of association strength as an a priori parameter that arises through semantic and linguistic experience (e.g., Wagner et al., 2001). In contrast, the previously used measure of association strength, agreement, is relative to alternative responses. Thus, if participants in the norming sample spread their responses fairly evenly between several strongly associated responses, the stimulus would be incorrectly classified as having low association strength when it in fact has both high association strength and high competition between alternative responses. Absolute LSA-based measures eliminate these problems with the previously used relative measures based on norming data, which makes purer, uncorrelated measures of both retrieval and selection demand possible. In addition, a new measure of competition (entropy, computed over LSA association values) reflects competition between all alternative responses rather than just the two most active responses. In behavioral studies, these purer, more theoretically justified measures of retrieval demands (LSA strength) and selection demands (LSA entropy) revealed effects of each factor on RTs controlling for the effect of the other factor, challenging accounts that posited a role for only selection or controlled retrieval (Snyder \& Munakata, 2008).

We have since replicated these behavioral results using a $2 \times 2$ design (high and low competition and high and low association strength), again finding strong effects of selection and controlled retrieval demands (Snyder et al., 2010). Moreover, this design allowed us to examine interactions between selection and retrieval demands, something prior studies had been unable as it was not possible to create a full $2 \times 2$ design with previously used measures of competition and association strength (Crescentini et al., 2010; Martin \& Cheng, 2006). Importantly, there was a significant interaction in the behavioral results: The effects of selection demand on RTs were greatest when retrieval demands were low. Our biologically plausible neural network model replicates and explains this interaction (Snyder et al., 2010). When it is easy to retrieve a response, activating multiple competing responses serves only to increase selection demands, slowing responding. However, when it is difficult to retrieve any response, spreading activation between multiple weakly associated responses (e.g., between open and close when generating a response for folder) increases the activation level of all responses, aiding retrieval, and partially offsetting selection costs.

Thus, the ability to respond in underdetermined situations is affected by both selection and retrieval demands, and these processes interact in meaningful ways. These findings highlight the need for unconfounded measures of selection and retrieval demands in studies of localization of neural function, which is a major goal of the current study. There are two general possible neural implementations of this pattern of behavioral results. First, separate neural substrates could support selection and controlled retrieval-either different subregions of left VLPFC, as proposed by the two-process account (Badre \& Wagner, 2007), or one process in left VLPFC and one elsewhere, as proposed by the selection (e.g., Thompson-Schill et al., 1997) and controlled retrieval (e.g., Martin \& Cheng, 2006) accounts. Alternatively, shared neural substrates (e.g., left VLPFC) could support selection and controlled retrieval through different mechanisms. For instance, our neural network model suggests that competitive lateral inhibition is specifically critical for selection whereas recurrent connectivity supports both retrieval and selection (Snyder et al., 2010). Moreover, if a common neural 
substrate supports both selection and controlled retrieval, then the activation pattern of left LVPFC should also show the interaction seen behaviorally, such that selection effects are stronger when retrieval demands are low.

In the current study, we determine the neural substrates important for selection and controlled retrieval. By using unconfounded LSA-based measures of competition and association strength and a full $2 \times 2$ design, we are able to test for main and interactive effects of selection and retrieval demands on brain activity for the first time in a neuroimaging study. We demonstrate that the same regions of left VLPFC support both selection and controlled retrieval, contrary to previous theories. Moreover, selection and controlled retrieval interact in left VLPFC, with larger selection effects when retrieval demands are low.

\section{METHODS}

\section{Subjects}

Eighteen healthy, right-handed, young adults ( 9 women) from the University of Colorado community participated in this study. Three additional subjects participated but were excluded from analysis because of excessively high error rates $(>25 \%)$. In addition, one outlier was excluded from analysis. ${ }^{2}$ All subjects were native English speakers, had no history of neurological conditions or head injury, and were not taking any psychoactive medication. Subjects gave informed consent and were treated in accordance with procedures approved by the University of Colorado Institutional Review Board.

\section{Design and Stimuli}

Stimuli were 100 nouns in a $2 \times 2$ design: High and low association strength between nouns and possible verb responses (retrieval demand) crossed with high and low competition among alternative responses (selection demand), with 25 trials/condition (Figure 1). Association strength was calculated as in previous work (Snyder et al., 2010; Snyder \& Munakata, 2008) using LSA (Landauer et al., 1998). LSA extracts the similarity of words, capturing contextual as well as co-occurrence information, and captures human semantic knowledge and behavior (e.g., Tse \& Altarriba, 2007; Wolfe \& Goldman, 2003; Dunn, Almeida, Barclay, Waterreus, \& Flicker, 2002; Landauer et al., 1998). The "general reading up to first-year college" corpus was used, and a term-to-term (nouns to verbs) comparison was used to obtain the LSA cosine (association strength) between the nouns and all verb responses generated by two or more subjects in a separate norming sample $(n=$ 50). Association strength was calculated as the average of three measures: (a) the strongest LSA cosine, (b) the LSA cosine for the most frequent response given by the norming sample, and (c) a weighted average of the LSA cosines for all verb responses given by the norming sample. Competition was defined as entropy $(H=-\Sigma(p(i) * \ln p(i))$,

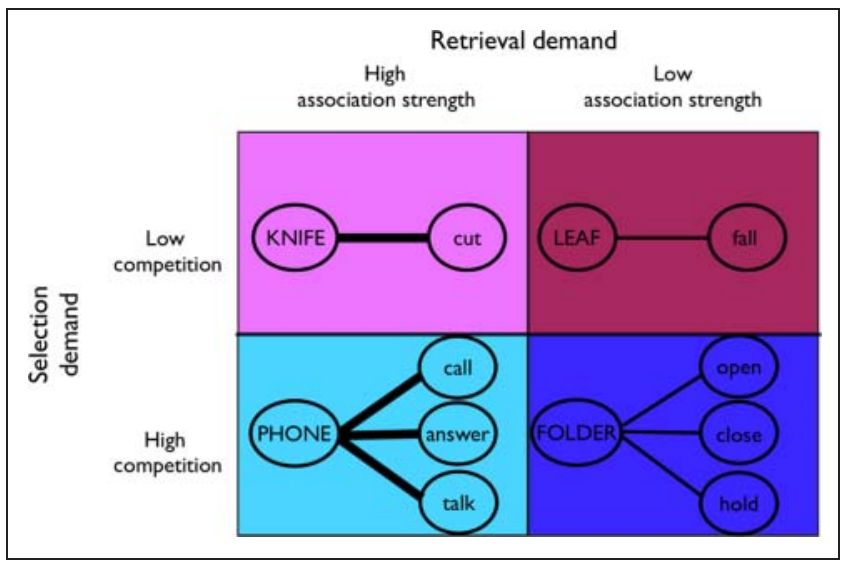

Figure 1. Verb generation task design with example items. Selection demand (high vs. low competition) is crossed with retrieval demand (high vs. low association strength). Nouns in the high selection demand conditions have multiple possible verb responses, whereas nouns in the low selection demand conditions have few possible verb responses (quantified as the LSA entropy, see Methods). Nouns in the high retrieval demand conditions have only weakly associated verb responses, whereas nouns in the low retrieval demand conditions have strongly associated verb responses (quantified as the LSA cosine, see Methods). High and low selection demand conditions are matched on retrieval demand, and high and low retrieval demand conditions are matched on selection demand, allowing the effects of each to be examined.

wherein $p(i)$ is the cosine between the stimulus and each alternative response, divided by the sum of LSA cosines among all alternative responses (Snyder \& Munakata, 2008). Therefore, entropy is zero when there is only one response (e.g., the cosine is 1) and increases as additional responses are equally associated with the stimuli (Snyder $\&$ Munakata, 2008). High and low association strength conditions were matched on LSA entropy, whereas high and low competition conditions were matched on association strength, allowing unconfounded effects of each variable to be assessed. ${ }^{3}$ Stimuli are the same as those in Snyder et al. (2010), and behavioral data from a separate, large sample are reported in full in Snyder et al. (2010). The full stimulus set is available from the authors upon request.

\section{Procedure}

Subjects were instructed to generate the first verb that came to mind when presented with a noun stimulus (e.g., "cat"). The verb could be either something the noun does (e.g., "meow") or something you do with it (e.g., "feed"). Subjects were given an example and eight practice trials before entering the scanner and were reminded of the instructions before beginning the task. During image acquisition, subjects completed 25 trials in each condition for a total of 100 trials. On each trial, subjects view a fixation point for $500 \mathrm{msec}$, followed by a noun cue for $3500 \mathrm{msec}$, and respond by saying a verb associated with the noun. Verbal responses are collected with a fiber optic noisecanceling microphone (Optoacoustics Ltd., Or-Yuhuda, Israel) via a procedure that has been found to minimize 
head motion (Barch, Sabb, Carter, Braver, \& Noll, 1999). A rapid event-related paradigm was used: The sequence was optimized using Optseq (surfer.nmr.mgh.harvard.edu/ fswiki/optseq2), including 50 null events (fixations) with a log jitter to maximize power. Presentation of items from each condition was intermixed with first-order counterbalancing. Within-condition item order was randomized across subjects. Data were acquired in one functional run, lasting about 9 min.

\section{Image Acquisition and Processing}

Data were acquired with a 3-T GE Signal whole-body MRI scanner at the University of Colorado Health Sciences Center, using T2*-weighted echo EPI [repetition time $=$ $2000 \mathrm{msec}$, echo time $=32 \mathrm{msec}$, flip angle $=70^{\circ}$ ]. Functional data were collected in a single run of 258 EPI volumes, each consisting of 32 slices (slice thickness $=4 \mathrm{~mm}$, gap $=0 \mathrm{~mm}$, field of view $=220 \mathrm{~mm}$, in-plane matrix $=$ $64 \times 64$, in-plane resolution $=3.44 \times 3.44 \mathrm{~mm}^{2}$ ), angled parallel to the AC-PC line. Before the functional run, highresolution T1-weighted 3-D Inversion Recovery (IR)Spoiled Gradient Echo (SPGR) full-head anatomical images were acquired along the coronal plane (124 slices, slice thickness $=1.7 \mathrm{~mm}$, repetition time $=9 \mathrm{msec}$, echo time $=$ $2 \mathrm{msec}$, flip angle $=10^{\circ}$, inversion time $=500 \mathrm{msec}$, field of view $=220 \mathrm{~mm}$, matrix $=256 \times 256$, in-plane resolution $=0.87 \mathrm{~mm} \times 0.87 \mathrm{~mm}$ ). The scanner was equipped with a standard head coil, and participants' heads were secured with moldable pillows to minimize head motion. Stimuli were displayed through fiber optic goggles, and participants respond by speaking into a fiber optic noisecanceling microphone (Optoacoustics Ltd., Or-Yuhuda, Israel) positioned directly above their mouth. All participants met our criteria for minimal head motion $(<2 \mathrm{~mm}$ translation $/ 2^{\circ}$ rotation in any direction).

Image preprocessing and analysis were largely conducted with FSL (FMRIB's Software Library). After discarding the first six volumes of the run to allow the MRI signal to reach steady state, the remaining images in each participant's time series were motion corrected using MCFLIRT (Jenkinson \& Smith, 2001), and nonbrain voxels were removed using BET. Images in the data series were spatially smoothed with a 3-D Gaussian kernel (FWHM $=8 \mathrm{~mm}$ ), intensity normalized for all volumes by the same factor, and high-pass filtered to remove high-frequency noise $(\sigma=100 \mathrm{sec})$. After statistical analysis for each participant's time series, the statistical maps (reflecting each participant's response in each condition) were normalized into the common MNI-152 stereotaxic space, using FLIRT (FMRIB's Linear Image Registration Tool; Jenkinson, Bannister, Brady, \& Smith, 2002) before random effect group analyses were performed. Subsequent statistical analyses were conducted using FEAT (FMRIB's Easy Analysis Tool). General linear model analyses of the fMRI time series data were conducted and then subjected to grouplevel random effects analysis.

\section{RESULTS}

\section{Behavioral Results}

RT data were analyzed with a $2 \times 2$ repeated measures ANOVA. Replicating previous findings (Snyder et al., 2010), participants were slowed by greater competition (greater selection demand, $F(1,16)=119.16, p<.001)$ and lower association strength (greater retrieval demand, $F(1,16)=578.80, p<.001) .{ }^{4}$ Specifically, RTs were longer in the high competition ( $\log \mathrm{RT}, M=7.70, S E=0.02)$ than low competition (log RT, $M=7.62, S E=0.02$ ) conditions and longer in the low association strength (log RT, $M=7.72, S E=0.02)$ than high association strength (log RT, $M=7.60, S E=0.02$ ) conditions. Also consistent with previous results, the effects of competition (selection costs) were numerically higher under high association strength (low retrieval demands; log RT difference, $M=0.10, S E=0.01)$ than under low association strength (high retrieval demands; $\log$ RT difference, $M=0.07$, $S E=0.01)$, although the interaction did not reach significance given the small number of subjects $(F(1,16)=$ $1.82, p=.197)$.

\section{Left VLPFC Region of Interest Analyses}

ROI analyses were conducted for the key regions hypothesized to play a role in controlled retrieval and selection: left anterior VLPFC and left mid-VLPFC. Spherical ROIs were defined around the mean coordinates identified in Badre and Wagner (2007) for left anterior VLPFC $(-48,30,-6)$ and left mid-VLPFC $(-50,25,14),{ }^{5}$ with a radius of $10 \mathrm{~mm}$. Activation for each condition versus fixation baseline within each ROI was extracted for each participant and subjected to a $2 \times 2 \times 2$ (Competition $\times$ Association Strength $\times$ Region) repeated measures ANOVA. There was a significant main effect of Competition (selection demand), with greater activation in the high competition than low competition conditions $(F(1,16)=15.32$, $p=.001)$, and a significant main effect of Association Strength (retrieval demand), with greater activation in the low association strength than high association strength conditions $(F(1,16)=21.53, p<.001)$. There was also a Significant Competition $\times$ Association Strength interaction: Effects of Competition were greatest when Association Strength was high (low retrieval demand; $F(1,16)=4.68$, $p=.046)$. There was no main effect of Region $(F(1,16)=$ $0.27, p=.6)$. Importantly, there were no interactions with Region (Region $\times$ Competition $F(1,16)=0.72$, $p=.4$; Region $\times$ Association Strength $F(1,16)=0.95$, $p=.3$; Region $\times$ Competition $\times$ Association Strength $F(1,16)=0.09, p=.8)$. Within each VLPFC ROI, main effects of Competition and Association Strength were significant and the interaction between Competition and Association Strength was marginal, as confirmed by $2 \times 2$ (Competition $\times$ Association Strength) ANOVAs run for anterior and mid-VLPFC ROIs separately (anterior VLPFC: Competition $F(1,16)=8.47, p=.01$, Association 
Strength $F(1,16)=16.11, p=.001$, Competition $\times$ Association Strength interaction $F(1,16)=3.60, p=.076$; mid-VLPFC: Competition $F(1,16)=14.92, p=.001$, Association Strength $F(1,16)=22.39, p<.001$, Compe-

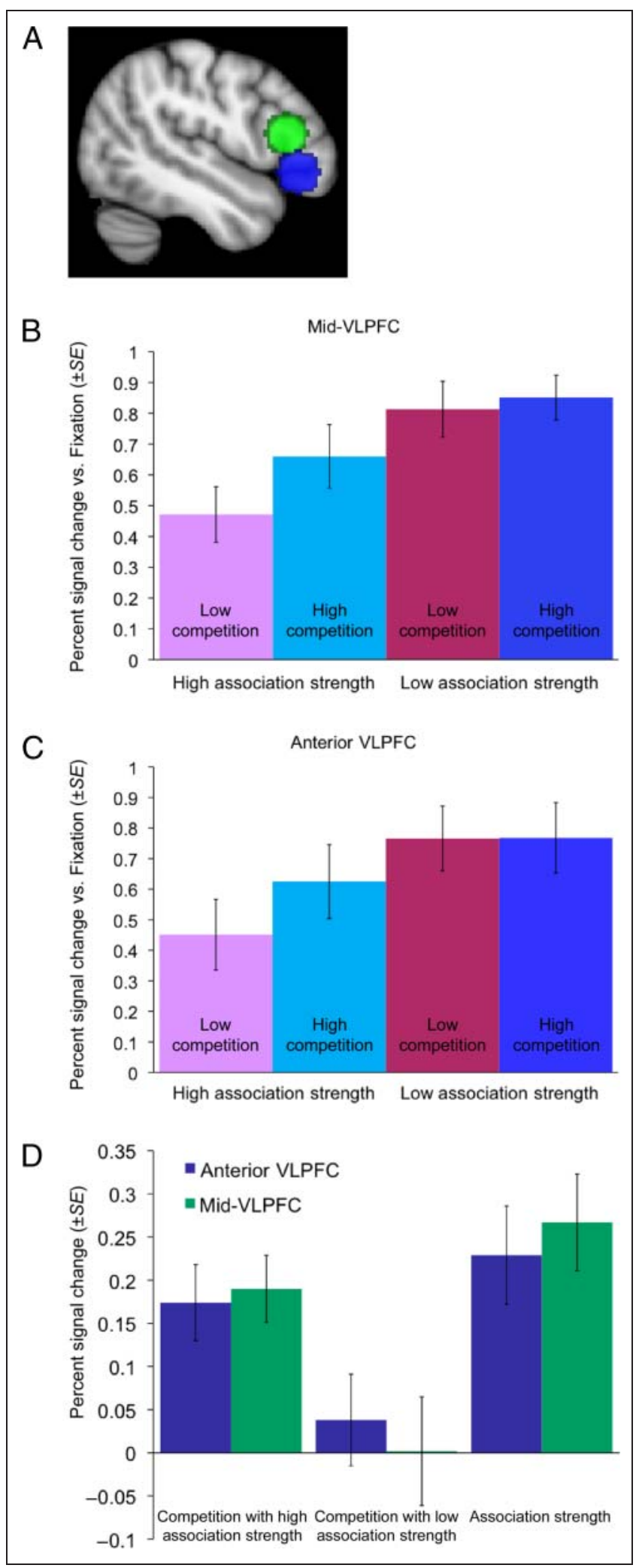

tition $\times$ Association Strength interaction $F(1,16)=4.39$, $p=.052) .{ }^{6}$ Thus, the left anterior and mid-VLPFC ROIs showed similar patterns of activity (Figure 2).

\section{Whole-brain Analysis}

In addition, exploratory whole-brain analyses were conducted for the following key contrasts: (1) high versus low association strength, collapsing across levels of competition (controlled retrieval), (2) high versus low competition, collapsing across levels of association strength (selection), (3) high versus low competition with high association strength (selection with low retrieval demand), and (4) high versus low competition with low association strength (selection with high retrieval demand; see Table 1 and Figure 3). In addition to mid (BA 45) and anterior (BA 47) left VLPFC, both competition and association strength manipulations engaged a larger frontal network, prominently including the pre-SMA in the superior frontal gyrus and right VLPFC. As in the ROI analysis, competition effects are most apparent when association strength is high (retrieval demands are low). The association strength manipulation additionally recruited a wide network of other medial and lateral PFC areas. In addition, both competition and association strength manipulations activated posterior cortical areas, including temporal and occipital cortex.

\section{DISCUSSION}

The current study used LSA-based measures to unconfound competition and association strength, which revealed main and interactive effects of selection and retrieval demand on activation of left VLPFC. Specifically, left VLPFC was more active when there was high competition between alternative responses (revealing an effect of selection demand) and when possible responses were weakly associated with the noun cue (revealing an effect of retrieval demand). Moreover, consistent with previous behavioral findings (Snyder et al., 2010), selection and controlled retrieval interact in left VLPFC, with increased activation in this region increasing with greater demands on selection,

Figure 2. VLPFC ROI activation. (A) ROIs were defined in anterior VLPFC (blue) and mid-VLPFC (green). Both mid-VLPFC (B) and anterior VLPFC (C) are sensitive to both competition (selection demand) and association strength (retrieval demands), and competition effects are strongest when association strength is high (retrieval demands are low). Mid-VLPFC and anterior VLPFC respond similarly (D), with no significant condition by region interactions. (D) Percent signal change for each key contrast: competition with high association strength $=$ high competition/high association strength low competition/high association strength; competition with low association strength $=$ high competition/low association strength low competition/low association strength; association strength = low association strength - high association strength). 
Table 1. Peak Voxel Coordinates, Anatomical Locations, and Approximate Brodmann's Areas from Exploratory Whole-brain Random Effects Analysis

\begin{tabular}{|c|c|c|c|c|c|c|c|c|}
\hline Contrast & & Region & $B A$ & $\operatorname{Max} Z$ & $\begin{array}{l}\text { No. of } \\
\text { Voxels }\end{array}$ & $x$ & $y$ & $z$ \\
\hline \multirow{9}{*}{$\begin{array}{l}\text { Selection (high competition > } \\
\text { low competition, collapsing } \\
\text { across levels of } \\
\text { association strength) }\end{array}$} & \multicolumn{2}{|c|}{ Superior frontal gyrus (L) } & 6 & 3.30 & 181 & -6 & 16 & 60 \\
\hline & \multicolumn{2}{|c|}{ Inferior frontal gyrus (VLPFC) (L) } & 45 & 3.24 & 127 & -44 & 20 & 14 \\
\hline & \multicolumn{2}{|c|}{ Inferior frontal gyrus (VLPFC) (L) } & 47 & 3.07 & 103 & -30 & 26 & -16 \\
\hline & \multicolumn{2}{|c|}{ Inferior frontal gyrus (VLPFC) (R) } & 47 & 2.98 & 59 & 36 & 28 & -12 \\
\hline & \multicolumn{2}{|c|}{ Medial frontal gyrus (R) } & 10 & 2.88 & 33 & 10 & 56 & 12 \\
\hline & \multicolumn{2}{|c|}{ Middle temporal gyrus (L) } & 21 & 3.00 & 41 & -66 & -28 & -6 \\
\hline & \multicolumn{2}{|c|}{ Middle temporal gyrus (R) } & 21 & 3.15 & 36 & 56 & 10 & -36 \\
\hline & \multicolumn{2}{|c|}{ Inferior occipital gyrus (R) } & 17 & 3.01 & 42 & 16 & -92 & -8 \\
\hline & \multicolumn{2}{|l|}{ Lingual gyrus (L) } & 18 & 3.09 & 63 & -14 & -92 & -14 \\
\hline \multirow{10}{*}{$\begin{array}{l}\text { Selection with low retrieval } \\
\text { demands (high competition/ } \\
\text { high association strength }> \\
\text { low competition/high } \\
\text { association strength) }\end{array}$} & \multicolumn{2}{|c|}{ Inferior frontal gyrus (VLPFC) (L) } & 45 & 3.18 & 362 & -42 & 20 & 14 \\
\hline & \multicolumn{2}{|c|}{ Superior frontal gyrus (L) } & 6 & 3.17 & 245 & -6 & 14 & 60 \\
\hline & \multicolumn{2}{|c|}{ Inferior frontal gyrus (R) } & 47 & 3.23 & 68 & 42 & 26 & -24 \\
\hline & \multicolumn{2}{|c|}{ Inferior frontal gyrus (L) } & 47 & 3.01 & 21 & -56 & 28 & -6 \\
\hline & \multicolumn{2}{|c|}{ Parahippocampal gyrus (R) } & 35 & 3.03 & 94 & 16 & -32 & -16 \\
\hline & \multicolumn{2}{|c|}{ Inferior temporal gyrus (L) } & 37 & 3.09 & 75 & -52 & -52 & -4 \\
\hline & \multicolumn{2}{|c|}{ Middle temporal gyrus (L) } & 21 & 2.93 & 53 & -68 & -30 & -4 \\
\hline & \multicolumn{2}{|c|}{ Superior temporal gyrus (L) } & 38 & 3.23 & 254 & -42 & 24 & -26 \\
\hline & \multicolumn{2}{|l|}{ Brainstem } & $\mathrm{n} / \mathrm{a}$ & 3.11 & 289 & 30 & -74 & -30 \\
\hline & \multicolumn{2}{|l|}{ Brainstem } & $\mathrm{n} / \mathrm{a}$ & 3.06 & 81 & -26 & -58 & -34 \\
\hline $\begin{array}{l}\text { Selection with high retrieval } \\
\text { demands (high competition/ } \\
\text { low association strength }> \\
\text { low competition/low } \\
\text { association strength) }\end{array}$ & \multicolumn{2}{|c|}{ Medial frontal gyrus } & 10 & 2.80 & 29 & 10 & 54 & 18 \\
\hline \multirow{14}{*}{$\begin{array}{l}\text { Retrieval (low association } \\
\text { strength > high association } \\
\text { strength, collapsing across } \\
\text { levels of competition) }\end{array}$} & \multicolumn{3}{|c|}{ Large left/medial frontal cluster } & 5.38 & 11,947 & & & \\
\hline & \multirow[t]{13}{*}{ Local maxima $^{a}$} & Superior frontal gyrus (L) & 6 & 5.38 & & -6 & 14 & 60 \\
\hline & & Superior frontal gyrus (R) & 6 & 3.70 & & 4 & 4 & 64 \\
\hline & & Superior frontal gyrus (L) & 8 & 4.53 & & -4 & 24 & 54 \\
\hline & & Anterior cingulate (L) & 32 & 3.09 & & -2 & 38 & 26 \\
\hline & & Anterior cingulate $(\mathrm{R})$ & 24 & 3.03 & & 10 & 34 & 22 \\
\hline & & Cingulate gyrus (L) & 32 & 4.49 & & -8 & 18 & 38 \\
\hline & & Cingulate gyrus (R) & 32 & 3.18 & & 12 & 22 & 38 \\
\hline & & Inferior frontal gyrus (VLPFC) (L) & 45 & 4.87 & & -50 & 24 & 18 \\
\hline & & Inferior frontal gyrus (VLPFC) (L) & 47 & 4.54 & & -46 & 26 & -4 \\
\hline & & Middle frontal gyrus (L) & 6 & 4.50 & & -46 & 4 & 46 \\
\hline & & Middle frontal gyrus (L) & 9 & 4.25 & & -44 & 10 & 38 \\
\hline & & Middle frontal gyrus (L) & 11 & 2.90 & & -44 & 48 & -12 \\
\hline & & Medial frontal gyrus (L) & 6 & 5.03 & & -2 & 12 & 52 \\
\hline
\end{tabular}


Table 1. (continued)

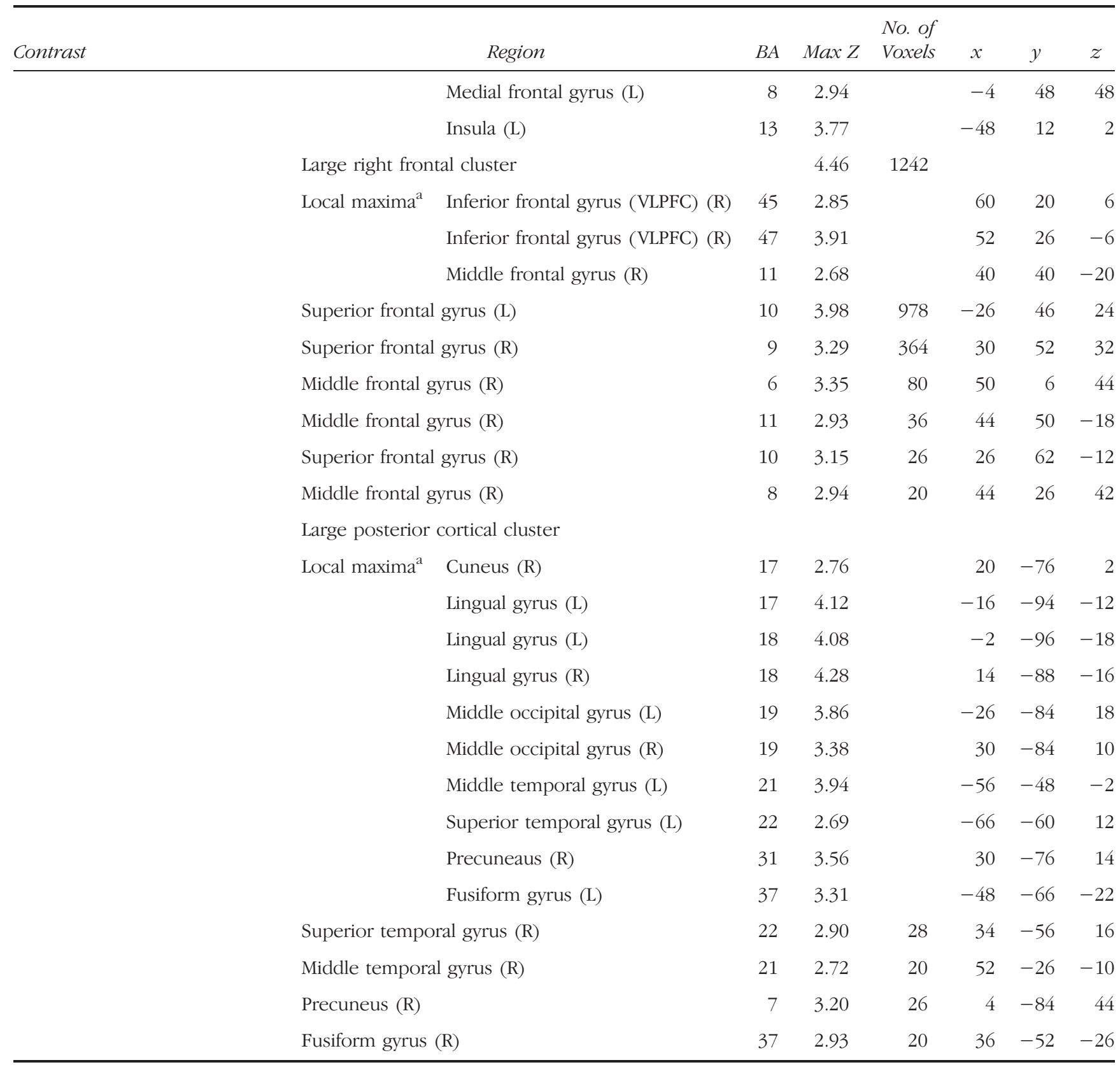

All clusters $z>2.58, p<.01$, two-tailed. BA = Brodmann's area; $\mathrm{L}=$ left; $\mathrm{R}=$ right; $\mathrm{n} / \mathrm{a}=$ not applicable.

${ }^{\mathrm{a}}$ When there was more than one local maximum in the same BA and hemisphere, the peak with the highest maximum $Z$ is reported.

when retrieval demands are low. When retrieval demands are high, selection demands did not modulate the observed activation of this region. It is possible that when retrieval demands are high, selection costs may be partially offset by the advantage multiple responses confer on retrieval (Snyder et al., 2010). Thus, it may be impossible to observe an effect of selection demands if retrieval demands are high, potentially explaining the null results for selection manipulations in some previous studies. This finding also suggests that future studies investigating selection processes should seek to minimize retrieval demands to increase power for observing selection effects.
Critically, in the current study, both mid-VLPFC and anterior VLPFC show nearly identical profiles, with no significant interactions between region and any task condition. Thus, the same regions of left VLPFC support both selection and controlled retrieval, and these processes interact. These results challenge previous accounts and may help explain mixed findings in the prior literature. Previous studies of selection and retrieval during language production used response-frequency-based measures that were highly correlated, such that conditions differing on one measure also differed on the other, confounding retrieval and selection demands. Two recent studies 
attempted to address this problem by creating high and low selection demand conditions matched on retrieval demand and high and low retrieval demand conditions matched on selection demand (e.g., Crescentini et al., 2010; Martin \& Cheng, 2006). However, attempting to separate highly collinear variables in this way tends to produce severe restrictions of range and, thus, low power and manipulation failures. Indeed, a reanalysis of Martin and Cheng's (2006) conditions with LSA-based measures revealed that the high and low selection demand conditions did not actually differ in competition (whereas the high and low retrieval demand conditions did differ in association strength), likely explaining the failure to find an effect of selection demand in this study (Snyder \& Munakata, 2008). It is likely that a similar manipulation failure oc- curred for retrieval demands in Crescentini et al. (2010), given the failure to find an effect of retrieval demand in the whole-brain analysis and unexpected results in the ROI analyses (greater VLPFC activation in the low retrieval demand condition for the noun generation task). ${ }^{7}$ Thus, previous attempts to disentangle to the effects of selection and retrieval demands have proved unsatisfactory. Using LSA-based measures to disentangle these factors, the results of the current study challenge previous theories that posit a single role of left VLPFC in either selection or retrieval or a functional dissociation between mid-VLPFC and anterior VLPFC.

Given that the current study found that shared neural substrates in VLPFC responded to both selection and retrieval demands, one could argue that these manipulations
Figure 3. Exploratory wholebrain analysis activation. In addition to left VLPFC, both association strength (retrieval demand; A) and competition (selection demand) with high association strength (low retrieval demand; B) activate wider prefrontal networks, whereas competition with low association strength (high retrieval demand; $C$ ) activates medial frontal cortex $(p<.05$, two-tailed). A conjunction analysis confirms that association strength and competition with high association strength manipulations activate shared areas of left VLPFC and anterior cingulate/pre-SMA (D). Thus, prefrontal areas recruited by association strength manipulations (retrieval demand) are also recruited by competition manipulations (selection demand), even when association strength is high (retrieval demands are low). See Table 1 for all significant areas of activation in the whole-brain random effects analysis.

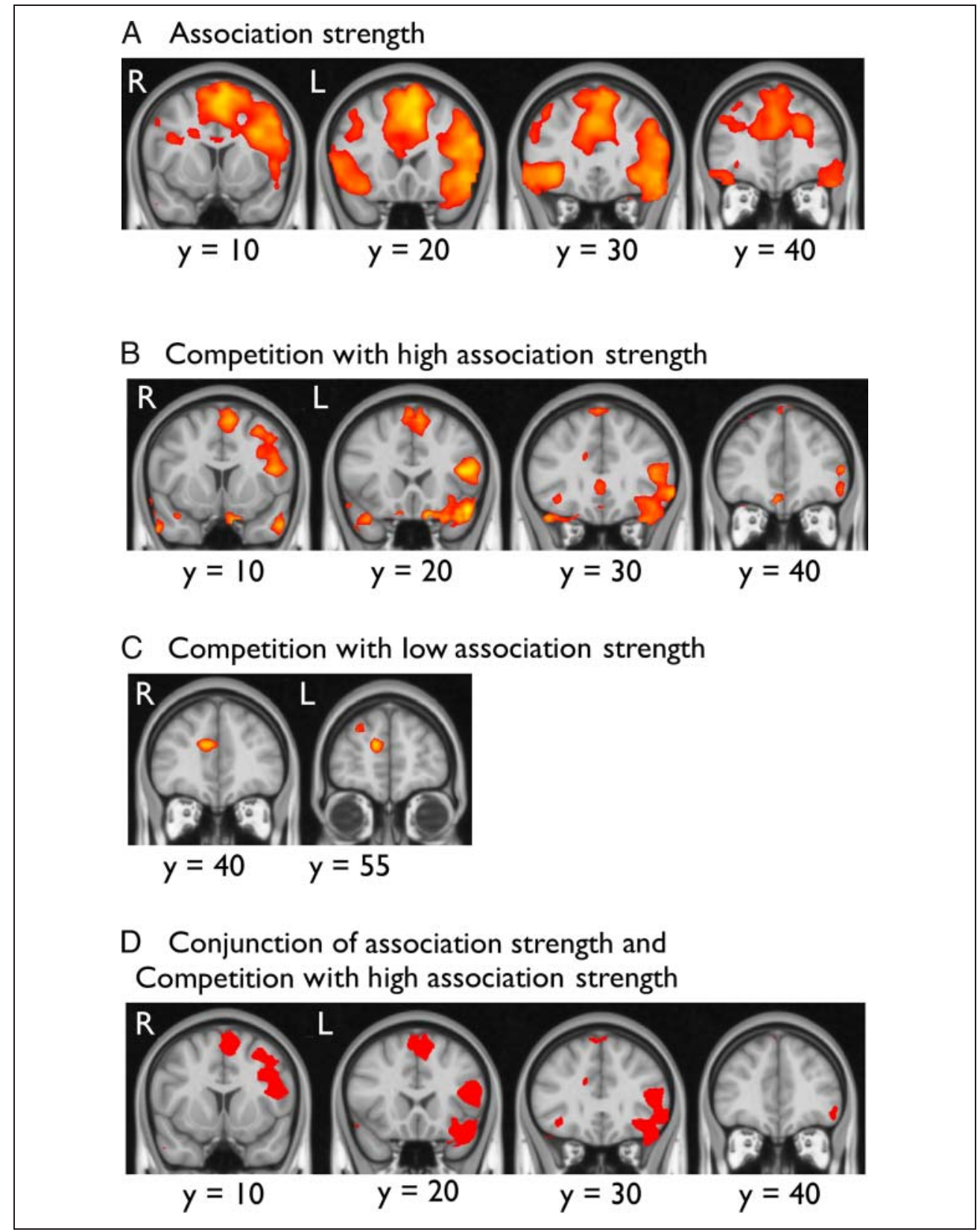


might affect a single process rather than separate selection and controlled retrieval processes. For example, it has been proposed that VLPFC activity is determined by a single memory activation value, which depends on the association strength between cues and items in memory, such that when multiple items are associated with the memory cue, the association strength of each is weakened by competition (Danker et al., 2008). Items with lower activation values are retrieved more slowly and require more control (and thus more VLPFC activity) than those with higher activation values (Danker et al., 2008). Although this account is consistent with results presented here, other evidence indicates that selection and controlled retrieval processes are separable (but interacting) at the level of neural mechanisms.

Specifically, as predicted by a neural network model of the verb generation task, selection and retrieval processes can be dissociated through the effects of neural inhibition (Snyder et al., 2010). Selection, but not controlled retrieval, depends on neural inhibition. During selection, competitive lateral inhibition, via GABAergic interneurons, may serve to suppress competing representations (Phillips \& Silverstein, 2003). When selection demands are high, populations of excitatory neurons representing multiple competing response options become active. These excitatory neurons in turn activate GABAergic interneurons, which then suppress the activity of other excitatory neurons with which they synapse. Because the most active excitatory neurons send more inhibition than they receive, this lateral inhibition serves to strengthen the most active representation and suppress competing representations, allowing one winning response to be selected. In contrast, the effects of retrieval demands are a direct consequence of the strength of the synaptic weights between stimuli (e.g., nouns) and their associated representations (e.g., verbs): Weaker weights cause a slower build-up of activation, requiring more time to reach the threshold to make a response (Snyder et al., 2010). In addition, we speculate that sustained neuronal activation, enabled by recurrent connections in PFC networks, may support retrieval of weakly active representations and may also contribute to selection by increasing the difference in activation levels between the most active representation and its competitors (that is, increasing the gain or signal-tonoise ratio; e.g., Snyder \& Munakata, 2008; Stedron, Sahni, \& Munakata, 2005; Hahnloser, Sarpeshkar, Mahowald, Douglas, \& Seung, 2000). As predicted from this framework in which neural inhibition affects selection but not retrieval, increasing GABAergic function through the GABA agonist midazolom leads to improvements in selection, whereas decreased GABAergic function associated with anxiety is linked to impairments in selection, with no effects on retrieval (Snyder et al., 2010). Thus, rather than favoring a single-process account, we posit that the same areas of left VLPFC support both selection and controlled retrieval through partially dissociable neural mechanisms.
Future work along (at least) three lines is needed to fully understand selection and retrieval processes for verbal as well nonverbal material. First, although mid-VLPFC and anterior VLPFC both contribute to selection and retrieval, they could potentially act on different types of representations of the same stimuli. For example, several theories posit a rostral-caudal gradient in PFC, with representations becoming more abstract in more anterior areas (e.g., Badre, 2008; Petrides, 2005). Thus, it is possible that anterior VLPFC retrieves and selects among more abstract semantic representations of the response options, whereas midVLPFC acts on less abstract (e.g., lexical) representations of the same responses. This possibility could be tested in future studies that manipulate the abstractness of the relevant representations. Second, the current study focused exclusively on selection and controlled retrieval during language production, and future work is needed to determine whether these findings extend to other domains.

Finally, although we have focused here on the role of competitive inhibitory dynamics and recurrent connectivity in the left VLPFC, it is probable that other brain regions and mechanisms also contribute to selection and controlled retrieval. Both processes clearly tap a larger network of brain areas, including other prefrontal regions and posterior cortical areas involved in representing semantic knowledge. Of particular interest, posterior dorsal ACC extending into the pre-SMA (pdACC/pre-SMA) was robustly activated by both selection and retrieval demands. In the cascade-of-control model (e.g., Banich, 2009; Milham \& Banich, 2005), this region is involved in guiding responding when earlier prefrontal processing areas have failed to exert adequate top-down control (see Silton et al., 2010, for ERP evidence). Although this model was developed based on evidence from the Stroop task and focused on interactions with dorsolateral PFC (rather than VLPFC), pdACC/pre-SMA may play a similar role during language production, as a final stage of control when left VLPFC has not been fully effective in retrieving or selecting words. Future research could test this possibility by investigating the temporal dynamics of the activation of left VLPFC and pdACC/pre-SMA, potentially through combined ERP/fMRI studies. In addition, future experimental and modeling work is needed to fully elucidate how these processes are neurally implemented. For example, $N$-Methyl-D-aspartate synapses may also play a crucial role in selection, because their voltage-dependent properties can implement a gain mechanism in which bottomup signals are selectively amplified, depending on their top-down saliency (e.g., Raffone, Murre, \& Wolters, 2003).

In summary, the findings of the current study enable a synthesis and reinterpretation of prior evidence and suggest that the ability to respond in underdetermined situations is affected by both selection and retrieval mechanisms subserved by left VLPFC, and these processes interact in meaningful ways. Although we are normally able to successfully deploy cognitive control mechanisms to retrieve responses and select between competing representations, 
this ability is compromised in a wide variety of clinical disorders, including ADHD (e.g., Tucha et al., 2005), anxiety and depression (e.g., Basso et al., 2007), autism (e.g., Kleinhans, Akshoomoff, \& Delis, 2005), schizophrenia (e.g., Moore, Savla, Woods, Jeste, \& Palmer, 2006), Huntington's disease (e.g., Troyer, Moscovitch, Winocur, Leach, \& Freedman, 1998; Randolph et al., 1993), and Parkinson's disease (e.g., Troyer et al., 1998). Better understanding these fundamental aspects of language production may ultimately have implications for better understanding and treatment of these deficits. Finally, beyond the domain of language, these findings may have broader implications for understanding the functional organization of PFC by illustrating that what have been conceptualized as distinct cognitive processes can be supported by shared neural substrates.

\section{Acknowledgments}

This research was supported by grants from the National Institutes of Health (P50-MH079485 and F31-MH087073). We thank Paula Villar, Kirsten Orcutt, and Luka Ruzic for assistance with data collection and analysis and members of the P50 center on Executive Function and Dysfunction, Cognitive Development Center, and Banich Lab for valuable discussions.

Reprint requests should be sent to Hannah R. Snyder, Department of Psychology and Neuroscience, University of Colorado, Muenzinger D244, 345 UCB, Boulder, CO 80309-0345, or via e-mail: hannah.snyder@colorado.edu.

\section{Notes}

1. There is debate about the underlying processes of selection and retrieval, with some researchers making theoretical arguments for a single process (e.g., Danker, Gunn, \& Anderson, 2008; Martin \& Byrne, 2006; Wagner et al., 2001). We, thus, primarily use the more theory-neutral terms competition and association strength when describing Methods and Results but also include the terms selection demands and retrieval demands throughout our article for consistency with the prior literature (e.g., Souza et al., 2009; Badre \& Wagner, 2007; Martin \& Cheng, 2006; Badre et al., 2005; Moss et al., 2005; Noppeney, Phillips, \& Price, 2004; Wagner et al., 2001; Thompson-Schill et al., 1997) and given the evidence that indicates separate processes (Snyder et al., 2010; see Discussion).

2. This subject showed an unusual pattern, with much higher activation in mid-VLPFC in the easiest experimental condition (low competition and high association strength Cook's D, $z=$ 2.75). The pattern of results remained the same when this subject was included, although power was slightly reduced. Analyses excluding the outlier are presented in the main text of Results, whereas results with the outlier included are presented in the footnotes.

3. One might ask whether association strength can truly be measured (or exist) independent of competition (e.g., Anderson \& Reder, 1999). For example, if when any given word occurs in the text corpus, it represents a case in which the word's synonyms were not used, does it decrease the association strengths of the synonyms with the co-occurring words in the text? This "push-pull" relationship between measures of association strength and competition would occur if our measures were based only on the co-occurrence of items in the text corpus and if alternative responses in our high selection demand conditions were mu- tually exclusive across paragraphs of text, but neither is the case. The association strength estimates derived from LSA are not simple contiguity frequencies, co-occurrence counts, or correlations in usage (Landauer et al., 1998). Rather, they capture contextual information (this is the "latent" part of the semantic analysis), such that words can be strongly associated even if they never directly co-occur together, so long as they occur in contexts with similar meanings (Landauer et al., 1998). That is, LSA can accurately estimate the association strength between word pairs never observed together by fitting them simultaneously in a higherdimensional semantic space (Landauer \& Dumais, 1997). In addition, alternative responses in the high selection demand conditions are not generally mutually exclusive synonyms but rather multiple actions associated with the noun. For example, talking about a cat purring in one sentence of a paragraph does not preclude talking about it licking in another sentence (or even within the same sentence), and because LSA learns associations over paragraphs rather than relying on simple contiguity, it would learn the association between cat and each of these verbs. Thus, our LSA-based measures do not involve an inherent tradeoff, such that nouns with multiple alternative verb responses have lower association strengths.

4. The pattern of results for the $2 \times 2$ behavioral data ANOVA remained the same with the outlier included, with significant main effects of competition $(F(1,17)=53.92, p<.001)$ and association strength $(F(1,17)=650.99, p<.001)$, and a nonsignificant interaction $(F(1,17)=1.43, p=.248)$.

5. These ROIs were chosen because they represent the mean coordinates from six previous studies of selection and controlled retrieval and are, thus, likely to be more reliable than coordinates from any individual study. To confirm that the results were not specific to the choice of ROI coordinates, additional analyses were conducted with anatomically defined ROIs for anterior VLPFC (left inferior gyrus pars orbitalis) and mid-VLPFC (left inferior gyrus pars triangularis) and yielded the same pattern of results. We, therefore, report only the coordinate-based ROIs, which represent a stronger test of the two-process account.

6. The pattern of results for all ANOVAs remained the same with the outlier included, with significant main effects of competition and association strength and a marginal interaction. $2 \times 2 \times 2$ ANOVA: Competition $(F(1,17)=12.10, p=.003)$, Association Strength $(F(1,17)=18.61, p<.001)$, Competition $\times$ Association Strength interaction $(F(1,17)=4.24, p=.055)$, with no interactions with region (Region $\times$ Competition $F(1,17)=0.04, p=.8$; Region $\times$ Association Strength $F(1,17)=1.73, p=.2$; Region $\times$ Competition $\times$ Association Strength $F(1,17)=0.20, p=.7)$. Anterior VLPFC: Competition $(F(1,17)=10.49, p=.005)$, Association Strength $(F(1,17)=12.04, p=.003)$, Competition $\times$ Association Strength interaction $(F(1,17)=3.55, p=.077)$. Mid-VLPFC: Competition $(F(1,17)=5.03, p=.038)$, Association Strength $(F(1,17)=22.18, p<.001)$, Competition $\times$ Association Strength interaction $(F(1,17)=3.61, p=.075)$.

7. A formal reanalysis with LSA-based measures cannot be carried out because the tasks were in Italian, for which there is currently no LSA corpus.

\section{REFERENCES}

Altmann, E. M., \& Gray, W. D. (2008). An integrated model of cognitive control in task switching. Psychological Review, 115, 602-639.

Anderson, J. R., \& Reder, L. M. (1999). Process, not representation: Reply to Radvansky (1999). Journal of Experimental Psychology: General, 128, 207-210.

Badre, D. (2008). Cognitive control, hierarchy, and the rostrocaudal organization of the frontal lobes. Trends in Cognitive Sciences, 12, 193-200. 
Badre, D., Poldrack, R. A., Paré-Blagoev, E. J., Insler, R. Z., \& Wagner, A. D. (2005). Dissociable controlled retrieval and generalized selection mechanisms in ventrolateral prefrontal cortex. Neuron, 47, 907-918.

Badre, D., \& Wagner, A. D. (2006). Computational and neurobiological mechanisms underlying cognitive flexibility. Proceedings of the National Academy of Sciences, U.S.A., 103, 7186-7191.

Badre, D., \& Wagner, A. D. (2007). Left ventrolateral prefrontal cortex and the cognitive control of memory. Neuropsychologia, 45, 2883-2901.

Banich, M. T. (2009). Executive function: The search for an integrated account. Current Directions in Psychological Science, 18, 89-94.

Barch, D. M., Braver, T. S., Sabb, F. W., \& Noll, D. C. (2000). Anterior cingulate and the monitoring of response conflict: Evidence from an fMRI study of overt verb generation. Journal of Cognitive Neuroscience, 12, 298-309.

Barch, D. M., Sabb, F. W., Carter, C. S., Braver, T. S., \& Noll, D. C. (1999). Overt verbal responding during fMRI scanning Empirical investigations of problems and potential solutions. Neuroimage, 10, 642-657.

Basso, M., Lowery, N., Ghormley, C., Combs, D., Purdie, R., Neel, J., et al. (2007). Comorbid anxiety corresponds with neuropsychological dysfunction in unipolar depression. Cognitive Neuropsychiatry, 12, 437-456.

Botvinick, M. M., Braver, T. S., Barch, D. M., Carter, C. S., \& Cohen, J. D. (2001). Conflict monitoring and cognitive control. Psychological Review, 108, 624-652.

Chou, T.-L., Booth, J. R., Bitan, T., Burman, D. D., Bigio, J. D., Cone, N. E., et al. (2006). Developmental and skill effects on the neural correlates of semantic processing to visually presented words. Human Brain Mapping, 27, 915-924.

Chou, T.-L., Booth, J. R., Burman, D. D., Bitan, T., Bigio, J. D., Lu, D., et al. (2006). Developmental changes in the neural correlates of semantic processing. Neuroimage, 29, $1141-1149$

Chou, T.-L., Chen, C.-W., Wu, M.-Y., \& Booth, J. R. (2009). The role of inferior frontal gyrus and inferior parietal lobule in semantic processing of Chinese characters. Experimental Brain Research, 198, 465-475.

Crescentini, C., Shallice, T., \& Macaluso, E. (2010). Item retrieval and competition in noun and verb generation: An fMRI study. Journal of Cognitive Neuroscience, 22, $1140-1157$.

Danker, J. F., Gunn, P., \& Anderson, J. R. (2008). A rational account of memory predicts left prefrontal activation during controlled retrieval. Cerebral Cortex, 18, 2674-2685.

Duncan, J., \& Owen, A. M. (2000). Common regions of the human frontal lobe recruited by diverse cognitive demands. Trends in Neurosciences, 23, 475-483.

Dunn, J. C., Almeida, O. P., Barclay, L., Waterreus, A., \& Flicker, L. (2002). Latent semantic analysis: A new method to measure prose recall. Journal of Clinical and Experimental Neuropsychology, 24, 26-35.

Gold, B., Balota, D., Jones, S., Powell, D., Smith, C., \& Andersen, A. (2006). Dissociation of automatic and strategic lexicalsemantics: Functional magnetic resonance imaging evidence for differing roles of multiple frontotemporal regions. Journal of Neuroscience, 26, 6523-6532.

Hahnloser, R. H. R., Sarpeshkar, R., Mahowald, M. A., Douglas, R. J., \& Seung, H. S. (2000). Digital selection and analogue amplification coexist in a cortex-inspired silicon circuit. Nature, 405, 948-951.

Jenkinson, M., Bannister, P., Brady, M., \& Smith, S. (2002). Improved optimization for the robust and accurate linear registration and motion correction of brain images. Neuroimage, 17, 825-841.
Jenkinson, M., \& Smith, S. (2001). A global optimisation method for robust affine registration of brain images. Medical Image Analysis, 5, 143-156.

Kan, I. P., Kable, J. W., Van Scoyoc, A., Chatterjee, A., \& Thompson-Schill, S. L. (2006). Fractionating the left frontal response to tools: Dissociable effects of motor experience and lexical competition. Journal of Cognitive Neuroscience, $18,267-277$

Kan, I. P., \& Thompson-Schill, S. L. (2004). Selection from perceptual and conceptual representations. Cognitive, Affective \& Behavioral Neuroscience, 4, 466-482.

Kleinhans, N., Akshoomoff, N., \& Delis, D. C. (2005). Executive functions in autism and Asperger's disorder: Flexibility, fluency, and inhibition. Developmental Neuropsychology, 27, 259-284

Landauer, T. K., \& Dumais, S. T. (1997). A solution to Plato's problem: The latent semantic analysis theory of acquisition, induction, and representation of knowledge. Psychological Review, 104, 211-240.

Landauer, T. K., Foltz, P. W., \& Laham, D. (1998). An introduction to latent semantic analysis. Discourse Processes, 25, 259-284.

Martin, R. C., \& Byrne, M. (2006). Why opening a door is as easy as eating an apple: A reply to Thompson-Schill and Botvinick (2006). Psychonomic Bulletin \& Review, 13, 400-411.

Martin, R. C., \& Cheng, Y. (2006). Selection demands versus association strength in the verb generation task Psychonomic Bulletin \& Review, 13, 396-401.

Milham, M., \& Banich, M. T. (2005). Anterior cingulate cortex: An fMRI analysis of conflict specificity and functional differentiation. Human Brain Mapping, 25, 328-335.

Miller, E. K. (2000). The prefrontal cortex and cognitive control. Nature Reviews Neuroscience, 1, 59-65.

Miyake, A., Emerson, M. J., Padilla, F., \& Ahn, J. C. (2004). Inner speech as a retrieval aid for task goals: The effects of cue type and articulatory suppression in the random task cuing paradigm. Acta Psychologica, 115, 123-142.

Moore, D. J., Savla, G. N., Woods, S. P., Jeste, D. V., \& Palmer, B. W. (2006). Verbal fluency impairments among middle-aged and older outpatients with schizophrenia are characterized by deficient switching. Schizophrenia Research, 87, 254-260.

Moss, H. E., Abdallah, S., Fletcher, P., Bright, P., Pilgrim, L., Acres, K., et al. (2005). Selecting among competing alternatives: Selection and retrieval in the left inferior frontal gyrus. Cerebral Cortex, 15, 1723-1735.

Nagel, I. E., Schumacher, E. H., Goebel, R., \& D’Esposito, M. (2008). Functional MRI investigation of verbal selection mechanisms in lateral prefrontal cortex. Neuroimage, 43, 801-807.

Nelson, J. K., Reuter-Lorenz, P. A., Persson, J., Sylvester, C.-Y. C., \& Jonides, J. (2009). Mapping interference resolution across task domains: A shared control process in left inferior frontal gyrus. Brain Research, 1256, 92-100.

Noppeney, U., Phillips, J., \& Price, C. J. (2004). The neural areas that control the retrieval and selection of semantics. Neuropsychologia, 42, 1269-1280.

Persson, J., Sylvester, C.-Y. C., Nelson, J. K., Welsh, K. M., Jonides, J., \& Reuter-Lorenz, P. A. (2004). Selection requirements during verb generation: Differential recruitment in older and younger adults. Neuroimage, 23, 1382-1390.

Petrides, M. (2005). Lateral prefrontal cortex: Architectonic and functional organization. Philosphical Transactions of the Royal Society of London, Series B, Biological Sciences, 360, 781-795.

Phillips, W. A., \& Silverstein, S. M. (2003). Convergence of biological and psychological perspectives on cognitive coordination in schizophrenia. Behavioral Brain Science, 26, 65-82. 
Raffone, A., Murre, J. M., \& Wolters, G. (2003). NMDA synapses can bias competition between object representations and mediate attentional selection. Behavioral and Brain Sciences, 26, 100-101.

Randolph, C., Bruan, A., Goldberg, T. E., \& Chase, T. (1993). Semantic fluency in Alzheimer's, Parkinson's and Huntington's disease: Dissociation of storage and retrieval failures. Neuropsychology, 7, 82-88.

Robinson, G., \& Cipolotti, L. (2004). Dynamic aphasia and the generation of language. Brain and Language, 91, 49-50.

Robinson, G., Shallice, T., \& Cipolotti, L. (2006). A failure of high level verbal response selection in progressive dynamic aphasia. Cognitive Neuropsychology, 22, 661-634.

Silton, R. L., Heller, W., Towers, D. N., Engels, A. S., Spielberg, J. M., Edgar, J. C., et al. (2010). The time course of activity in dorsolateral prefrontal cortex and anterior cingulate cortex during top-down attentional control. Neuroimage, 50, 1292-1302.

Snyder, H. R., Feigenson, K., \& Thompson-Schill, S. L. (2007). Prefrontal cortical response to conflict during semantic and phonological tasks. Journal of Cognitive Neuroscience, 19, 761-775.

Snyder, H. R., Hutchison, N., Nyhus, E., Curran, T., Banich, M. T., O'Reilly, R. C., et al. (2010). Neural inhibition enables selection during language processing. Proceedings of the National Academy of Sciences, U.S.A., 107, 16483-16488.

Snyder, H. R., \& Munakata, Y. (2008). So many options, so little time: The roles of association and competition in underdetermined responding. Psychonomic Bulletin $\&$ Review, 15, 1083-1088.

Souza, M., Donohue, S., \& Bunge, S. (2009). Controlled retrieval and selection of action-relevant knowledge mediated by partially overlapping regions of left ventrolateral prefrontal cortex. Neuroimage, 46, 299-307.

Stedron, J. M., Sahni, S. D., \& Munakata, Y. (2005). Common mechanisms for working memory and attention: The case of perseveration with visible solutions. Journal of Cognitive Neuroscience, 17, 623-631.

Thompson-Schill, S. L., \& Botvinick, M. M. (2006). Resolving conflict: A response to Martin and Cheng (2006). Psychonomic Bulletin E Review, 13, 402-408; discussion 409-411.
Thompson-Schill, S. L., D’Esposito, M., Aguirre, G. K., \& Farah, M. J. (1997). Role of left inferior prefrontal cortex in retrieval of semantic knowledge: A reevaluation. Proceedings of the National Academy of Sciences, U.S.A., 94, 14792-14797.

Thompson-Schill, S. L., Swick, D., Farah, M. J., D’Esposito, M., Kan, I. P., \& Knight, R. T. (1998). Verb generation in patients with focal frontal lesions: A neuropsychological test of neuroimaging findings. Proceedings of the National Academy of Sciences, U.S.A., 95, 15855-15860.

Tippett, L. J., Gendall, A., Farah, M. J., \& Thompson-Schill, S. L. (2004). Selection ability in Alzheimer's disease: Investigation of a component of semantic processing. Neuropsychology, 18, 163-173.

Tremblay, P., \& Gracco, V. L. (2006). Contribution of the frontal lobe to externally and internally specified verbal responses: fMRI evidence. Neuroimage, 33, 947-957.

Troyer, A. K., Moscovitch, M., Winocur, G., Leach, L., \& Freedman, M. (1998). Clustering and switching on verbal fluency tests in Alzheimer's and Parkinson's disease. Journal of the International Neuropsychological Society, 4, 137-143.

Tse, C. S., \& Altarriba, J. (2007). Testing the associative-link hypothesis in immediate serial recall: Evidence from word frequency and word imageability effects. Memory, 15, 675-690

Tucha, O., Mecklinger, L., Laufkötter, R., Kaunzinger, I., Paul, G., Klein, H., et al. (2005). Clustering and switching on verbal and figural fluency functions in adults with attention deficit hyperactivity disorder. Cognitive Neuropsychiatry, 10, 231-248.

Velanova, K., Jacoby, L. L., Wheeler, M. E., McAvoy, M. P., Petersen, S. E., \& Buckner, R. L. (2003). Functional-anatomic correlates of sustained and transient processing components engaged during controlled retrieval. Journal of Neuroscience, 23, 8460-8470.

Wagner, A. D., Paré-Blagoev, E. J., Clark, J., \& Poldrack, R. A. (2001). Recovering meaning: Left prefrontal cortex guides controlled semantic retrieval. Neuron, 31, 329-338.

Wolfe, M. B., \& Goldman, S. R. (2003). Use of latent semantic analysis for predicting psychological phenomena: Two issues and proposed solutions. Behavior Research Methods, Instruments, \& Computers, 35, 22-31. 